\title{
MMSE Filtering Performance of DH-AF Massive MIMO Relay Systems with Residual Transceiver Impairments
}

\author{
Anastasios K. Papazafeiropoulos*, Shree Krishna Sharma ${ }^{\dagger}$, and Symeon Chatzinotas ${ }^{\dagger}$ \\ *Communications and Signal Processing Group, Imperial College London, London, U.K. \\ ${ }^{\dagger}$ SnT - securityandtrust.lu, University of Luxembourg, Luxembourg \\ Email: a.papazafeiropoulos@imperial.ac.uk, \{shree.sharma, symeon.chatzinotas\}@uni.lu
}

\begin{abstract}
The emerging requirements of the fifth generation (5G) wireless communications are high spectral efficiency, low latency, and ubiquitous coverage. In this direction, Dual-Hop (DH) Amplify-and-Forward (AF) relaying has been widely investigated due to its simplicity, low implementation complexity and low transmission delay. However, most existing works assume ideal transceiver hardware which is impractical. In practice, a cost-efficient wireless transceiver has to combat the effects of several inevitable impairments such as high power amplifier nonlinearities, In-phase/Quadrature-phase (I/Q)-imbalance, and oscillator phase noise, which can be only partially compensated using calibration algorithms. In this direction, this paper analyzes the Minimum Mean Square Error (MMSE) filtering performance of a DH-AF Multiple-Input-Multiple-Output (MIMO) wireless system considering the effects of the residual additive impairments at the transmitter and receiver of both hops. Using free probability principles, the MMSE filtering performance of the considered system is studied and a tight lower bound is proposed by taking the effects of residual additive transceiver impairments into account. Our numerical results show that the MMSE filtering performance of the DH-AF massive MIMO relay system significantly degrades and results to saturation in the presence of residual additive transceiver impairments.
\end{abstract}

\section{INTRODUCTION}

Recently, relaying technology has received significant attention in wireless communications due to its several advantages such as coverage improvement and increased spectral efficiency [1]-[4]. Based on the mode of operation, the relaying strategies used for enhancing the signal levels in multihop cellular networks can be broadly categorized into Decode and Forward (DF), and Amplify and Forward (AF). This paper focuses on AF relaying, since it is of practical interest for future $5 \mathrm{G}$ wireless due to its several advantages such as simplicity, low implementation complexity and low transmission delay [1], [2].

Several works exist in the area of the performance analysis of Dual-Hop (DH) AF relay channels [3]-[5]. The contribution in [3] concerns the performance analysis of DH-AF MultipleInput-Multiple-Output (MIMO) relay channels by using finite dimensional random matrix theory. Specifically, the authors derived the expressions for the exact ergodic capacity, simplified closed-form expressions for the high Signal-to-Noise-Ratio (SNR) regime, and closed-form tight upper and lower bounds. Moreover, the authors in [4] studied the AF relaying in combination with collaborative base stations and determined the ergodic capacity for optimal and Minimum Mean Square Error (MMSE) receivers by employing a replica analysis. Furthermore, in [5], the MMSE filtering performance of a DHAF MIMO multiple-access channel was studied and a tight lower bound for the average MMSE was proposed by using free probability principles. However, all these works assumed ideal transceiver hardware, which is unrealistic in practice. In this regard, this paper focuses on the MMSE performance analysis of DH-AF MIMO relay channels by taking the effect of residual additive transceiver impairments into account.

Over the recent years, there has been an increasing interest in analyzing the effect of residual transceiver impairments on the performance of wireless communication systems. Besides the effects of channel fading, noise and the interference in wireless systems, inevitable hardware impairments arise such as high power amplifier nonlinearities [6], In-phase/Quadrature-phase (I/Q)-imbalance [7], and oscillator phase noise (PN) [8]. In particular, the effect of these impairments become more severe in systems having inexpensive hardware, which is one of the main requirements for a cost-efficient implementation of the massive MIMO technology. Although the performance degradation caused due to the aforementioned transceiver impairments can be partially mitigated by employing suitable calibration schemes at the transmitter or compensation algorithms at the receiver, a certain amount of distortion still remains due to residual hardware impairments [9]. Towards the direction of reducing this unaccounted distortion, several recent works have studied the effect of residual additive transceiver impairments on the system performance of both conventional and massive MIMO systems [9]-[12].

However, despite the significant importance of DH-AF relay systems in providing cost-efficient solutions for spectral efficiency enhancement and coverage expansion, only a few works have studied the effect of residual additive hardware impairments on these systems [13]-[15]. The contribution in [13] studied the effect of these impairments in DH-AF relay systems, however, the analysis was limited to the derivation of outage probability and simple capacity upper bounds. Recently, the authors in [15] analyzed the impact of hardware impairments on the performance of massive MIMO relay networks by using free probability theory, however, the performance metric was limited to ergodic capacity.

In this paper, we go one step beyond to carry out the 
performance analysis of DH-AF massive MIMO relay systems by investigating the MMSE filtering performance. More specifically, we extend the work in [5], which analyzed the MMSE filtering performance of a DH-AF MIMO multipleaccess channel, to the case of DH-AF massive MIMO relay systems with hardware impairments. Based on the principles of free probability, we derive a tight lower bound for the average MMSE by including the effects of residual hardware impairments. The derived average MMSE dictates the performance of single-user receivers after performing multiuser MMSE filtering.

The remainder of this paper is structured as follows: Section II introduces the system model of a DH-AF MIMO relay system with residual additive hardware impairments. Section III provides the theoretical analysis for the MMSE filtering performance of this system, while Section IV evaluates its performance by means of numerical results. Finally, Section V concludes the paper. Appendices follow with some preliminaries on random matrix theory and the proofs.

\section{SySTEM MODEL}

Initially, we consider a DH-AF MIMO relay system with ideal transceiver hardware. For the sake of simplicity, we assign the subscript 1 to the parameters between the source and the relay, while the parameters describing the second hop are indicated by the subscript 2. Based on the basic structure of a DH-AF system, partitioned by three nodes (the source, the relay, and the destination), we consider each node equipped with multiple antennas, in order to analyze the performance of large MIMO systems destined to be implemented in $5 \mathrm{G}$ wireless networks. Hence, a $K$-antennas source node, desiring to contact a distant $N$-antennas Base Station (BS), transmits first to an intermediate relay comprising an array of $M$ antennas (first hop). In other words, all the three nodes are compact stations equipped with multiple antennas, while the two hops are modeled through two point to point MIMO channels. In our model, the BS is assumed to be aware of the total system Channel State Information (CSI), while both the source and the relay have no CSI knowledge during their transmissions.

The channel model is expressed mathematically as

$$
\begin{aligned}
\mathbf{y}_{1} & =\mathbf{H}_{1} \mathbf{x}_{1}+\mathbf{z}_{1}, \\
\mathbf{y}_{2} & =\mathbf{H}_{2} \sqrt{\nu} \mathbf{y}_{1}+\mathbf{z}_{2} \\
& =\sqrt{\nu} \mathbf{H}_{2} \mathbf{H}_{1} \mathbf{x}_{1}+\sqrt{\nu} \mathbf{H}_{2} \mathbf{z}_{1}+\mathbf{z}_{2},
\end{aligned}
$$

where the source-relay and relay-BS input-output signal models exhibit Rayleigh fast-fading, expressed by Gaussian matrices with independent identically distributed (i.i.d.) complex circularly symmetric elements. Specifically, $\mathbf{H}_{1} \in \mathbb{C}^{M \times K} \sim$ $\mathcal{C N}\left(\mathbf{0}, \frac{1}{M} \mathbf{I}_{M} \otimes \mathbf{I}_{K}\right)$ is the channel matrix between the $K$ source antennas and the relay exhibiting flat-fading, while $\mathbf{H}_{2} \in \mathbb{C}^{N \times M} \sim \mathcal{C N}\left(\mathbf{0}, \frac{1}{N} \mathbf{I}_{N} \otimes \mathbf{I}_{M}\right)$ describes the channel matrix between the relay and the BS. Moreover, $\mathbf{x}_{1} \in \mathcal{C}^{K \times 1}$ is the Gaussian vector of symbols simultaneously transmitted by the source node with $\mathbb{E}\left[\mathbf{x}_{1} \mathbf{x}_{1}^{\mathrm{H}}\right]=\mathbf{Q}_{1}=\frac{\rho}{K} \mathbf{I}_{K}$, where $\rho$ is the total transmit power. In addition, $\mathbf{y}_{1}$ and $\mathbf{y}_{2}$ as well as $\mathbf{z}_{1} \sim \mathcal{C N}\left(\mathbf{0}, \mathbf{I}_{M}\right)$ and $\mathbf{z}_{2} \sim \mathcal{C N}\left(\mathbf{0}, \mathbf{I}_{N}\right)$ denote the received signals as well as the Additive White Gaussian Noise (AWGN) vectors at the relay and $\mathrm{BS}$, respectively. It is worthwhile to note that before forwarding the received signal $\mathbf{y}_{1}$ to the $\mathrm{BS}$, we consider that it is amplified by $\nu=\frac{\alpha}{M(1+\rho)}$, where we have placed a per relay-antenna fixed power constraint $\frac{\alpha}{M}$ with $\alpha$ being the total fixed gain of the relay, i.e., $\mathbb{E}\left[\left\|\sqrt{\nu} \mathbf{y}_{1}\right\|^{2}\right] \leq \alpha$. Herein, the expectation is taken over the signal, noise, and channel fading realizations.

Unfortunately, in reality, the transmitter (source), the relay, and the BS appear certain inevitable additive impairments such as I/Q imbalance, which cannot be mitigated completely [16], [17]. The model under study demands special attention because the relay plays two distinctive roles. Especially, it operates as a receiver during first hop, while it becomes the transmitter of the second hop. Taking this into consideration, in each node of the system, both a transmit and a receive impairment exist that cause a mismatch between the intended signal and what is actually transmitted during the transmit processing and a distortion of the received signal at the receiver side. More concretely, introducing the residual additive transceiver impairments to (1) and (2), we provide more realistic models for the respective links allowing us to investigate the impact of additive residual transceiver impairments on a DH-AF system.

In particular, we have:

$\mathbf{y}_{1}=\mathbf{H}_{1}\left(\mathbf{x}_{1}+\boldsymbol{\eta}_{\mathrm{t}_{1}}\right)+\boldsymbol{\eta}_{\mathrm{r}_{1}}+\mathbf{z}_{1}$,

$\mathbf{y}_{2}=\mathbf{H}_{2}\left(\sqrt{\nu} \mathbf{y}_{1}+\boldsymbol{\eta}_{\mathrm{t}_{2}}\right)+\boldsymbol{\eta}_{\mathrm{r}_{2}}+\mathbf{z}_{2}$

$=\sqrt{\nu} \mathbf{H}_{2} \mathbf{H}_{1}\left(\mathbf{x}_{1}+\boldsymbol{\eta}_{\mathrm{t}_{1}}\right)+\mathbf{H}_{2}\left(\sqrt{\nu}\left(\boldsymbol{\eta}_{\mathrm{r}_{1}}+\mathbf{z}_{1}\right)+\boldsymbol{\eta}_{\mathrm{t}_{2}}\right)+\boldsymbol{\eta}_{\mathrm{r}_{2}}+\mathbf{z}_{2}$,

where the additive terms $\boldsymbol{\eta}_{\mathrm{t} i}$ and $\boldsymbol{\eta}_{\mathrm{r} i}$ for $i=1,2$ are the distortion noises coming from the residual impairments in the transmitter and receiver of link $i$, respectively. Generally, the transmitter and the receiver distortion noises for the $i$ th link are modeled as Gaussian distributed, where their average power is proportional to the average signal power, as shown by measurement results [17]. The mathematical representations of these impairments are

$$
\begin{aligned}
\boldsymbol{\eta}_{\mathrm{t}_{i}} & \sim \mathcal{C N}\left(\mathbf{0}, \delta_{\mathrm{t}_{i}}^{2} \operatorname{diag}\left(q_{\mathrm{i}_{1}}, \ldots, q_{T_{\mathrm{i}}}\right)\right), \\
\boldsymbol{\eta}_{\mathrm{r}_{i}} & \sim \mathcal{C N}\left(\mathbf{0}, \delta_{\mathrm{r}_{i}}^{2} \operatorname{tr}\left(\mathbf{Q}_{i}\right) \mathbf{I}_{R_{\mathrm{i}}}\right)
\end{aligned}
$$

with $T_{\mathrm{i}}$ and $R_{\mathrm{i}}$ being the numbers of transmit and receive antennas of link $i$, i.e., $T_{1}=K, T_{2}=M$ and $R_{1}=M, R_{2}=N$, while $\mathbf{Q}_{i}$ is the transmit covariance matrix of the corresponding link with diagonal elements $q_{\mathrm{i}_{1}}, \ldots, q_{T_{\mathrm{i}}}{ }^{1}$. The levels of the residual impairments in the transmitter and the receiver of link $i$ are defined by the proportionality parameters $\delta_{\mathrm{t}_{i}}^{2}$ and $\delta_{\mathrm{r}_{i}}^{2}$. Given that the transmit covariance matrix of each link is different, regarding the first hop, we have

$$
\begin{aligned}
\boldsymbol{\eta}_{\mathrm{t}_{1}} & \sim \mathcal{C N}\left(\mathbf{0}, \delta_{\mathrm{t}_{1}}^{2} \frac{\rho}{K} \mathbf{I}_{K}\right), \\
\boldsymbol{\eta}_{\mathrm{r}_{1}} & \sim \mathcal{C N}\left(\mathbf{0}, \delta_{\mathrm{r}_{1}}^{2} \rho \mathbf{I}_{M}\right) .
\end{aligned}
$$

Similarly, since the input signal for the second hop is $\sqrt{\nu} \mathbf{y}_{i}$, the corresponding input covariance matrix is

$$
\begin{aligned}
\mathbf{Q}_{2}=\nu \mathbb{E}\left[\mathbf{y}_{1} \mathbf{y}_{1}^{\mathrm{H}}\right] & =\nu K\left(\mu+\delta_{\mathrm{t}_{1}}^{2} \mu+\delta_{\mathrm{r}_{1}}^{2} \mu+\frac{1}{K}\right) \mathbf{I}_{M} \\
& =\tilde{\mu} \nu K \mathbf{I}_{M},
\end{aligned}
$$

\footnotetext{
${ }^{1}$ The circularly-symmetric complex Gaussianity can be justified by the aggregate contribution of many impairments.
} 
where $\tilde{\mu}=\left(\mu+\delta_{\mathrm{t}_{1}}^{2} \mu+\delta_{\mathrm{r}_{1}}^{2} \mu+\frac{1}{K}\right)$. Note that now, $\nu=\frac{\alpha}{K M \tilde{\mu}}$, after accounting for fixed gain relaying. Thus, the additive transceiver impairments for the second hop take the form

$$
\begin{aligned}
\boldsymbol{\eta}_{\mathrm{t}_{2}} & \sim \mathcal{C N}\left(\mathbf{0}, \delta_{\mathrm{t}_{2}}^{2} \tilde{\mu} \nu K \mathbf{I}_{M}\right), \\
\boldsymbol{\eta}_{\mathrm{r}_{2}} & \sim \mathcal{C N}\left(\mathbf{0}, \delta_{\mathrm{r}_{2}}^{2} \tilde{\mu} \nu K M \mathbf{I}_{N}\right) .
\end{aligned}
$$

Obviously, the presence of impairments at the relay node signify two different distortion noises $\boldsymbol{\eta}_{\mathrm{r}_{1}}$ and $\boldsymbol{\eta}_{\mathrm{t}_{2}}$ as mentioned earlier. The latter one together with the distortion noise $\boldsymbol{\eta}_{\mathrm{r}_{2}}$ occurs at the BS and have been amplified during the second hop.

\section{MMSE Receiver Performance AnALysis}

In this section, we analyze the MMSE filtering performance of the DH-AF massive MIMO relay system by applying the tools from large random matrix theory. The ensuing asymptotic analysis, resorting to free probability theory, is beneficial because it leads quickly to deterministic results as the channel matrix dimensions (number of antennas) tend to infinity with a given ratio. However, it should be stressed that the results are valid even for finite number of antennas as depicted later in Section IV. For the DH-AF MIMO relay system, the average MMSE is expressed as

$$
\begin{aligned}
& \mathrm{MMSE}_{\mathrm{avg}}=\mathbb{E}\left[\frac{1}{K} \sum_{m=1}^{K} \mathrm{MMSE}_{m}\right] \\
& =\mathbb{E}\left[\frac{1}{K} \sum_{m=1}^{K}\left[\left(\mathbf{I}_{M}+\mu \nu \mathbf{H}_{1}^{\mathrm{H}} \mathbf{H}_{2}^{\mathrm{H}} \mathbf{R}^{-1} \mathbf{H}_{2} \mathbf{H}_{1}\right)^{-1}\right]_{k, k}\right] \\
& =\mathbb{E}\left[\frac{1}{K} \operatorname{tr}\left\{\left(\mathbf{I}_{M}+\mu \nu \mathbf{H}_{1}^{\mathrm{H}} \mathbf{H}_{2}^{\mathrm{H}} \mathbf{R}^{-1} \mathbf{H}_{2} \mathbf{H}_{1}\right)^{-1}\right\}\right] \\
& =\mathbb{E}\left[\frac { 1 } { K } \operatorname { t r } \left\{\left(\mathbf{I}_{N}+\tilde{f}_{2} \mathbf{H}_{2}\left(\mathbf{I}_{M}+\tilde{f}_{1} \mathbf{H}_{1} \mathbf{H}_{1}^{\mathrm{H}}\right) \mathbf{H}_{2}^{\mathrm{H}}\right)^{-1}\right.\right. \\
& \left.\left.\times\left(\mathbf{I}_{N}+\tilde{f}_{2} \mathbf{H}_{2}\left(\mathbf{I}_{M}+\tilde{f}_{3} \mathbf{H}_{1} \mathbf{H}_{1}^{\mathrm{H}}\right) \mathbf{H}_{2}^{\mathrm{H}}\right)\right\}\right],
\end{aligned}
$$

where $\mathbf{R}=\nu \mu \delta_{\mathrm{t}_{1}}^{2} \mathbf{H}_{2} \mathbf{H}_{1} \mathbf{H}_{1}^{\mathrm{H}} \mathbf{H}_{2}^{\mathrm{H}}+\nu\left(K \mu \delta_{\mathrm{r}_{1}}^{2}+1+\tilde{\mu} K \delta_{\mathrm{t}_{2}}^{2}\right)$ $\mathbf{H}_{2} \mathbf{H}_{2}^{\mathrm{H}}+\left(\nu \tilde{\mu} K M \delta_{\mathrm{r}_{2}}^{2}+1\right) \mathbf{I}_{N}, \quad \tilde{f}_{1}=\frac{\tilde{\delta}_{\mathrm{t}_{1}}^{2} \rho}{K\left(\tilde{\delta}_{\mathrm{t}_{2} \mathrm{r}_{1}}^{2}+\rho \delta_{\mathrm{t}_{2}}^{2} \tilde{\delta}_{\mathrm{t}_{1} \mathrm{r}_{1}}^{2}\right)}$, $\tilde{f}_{2}=\frac{\alpha\left(\tilde{\delta}_{\mathrm{t}^{2} \mathrm{r}_{1}}^{2}+\rho \delta_{\mathrm{t}_{2}}^{2} \tilde{\delta}_{1_{1} \mathrm{r}_{1}}^{2}\right)}{M \tilde{\delta}_{\mathrm{r}_{2}}^{2}\left(1+\rho \tilde{\delta}_{\mathrm{t}_{1} \mathrm{r}_{1}}^{2}\right)}$, and $\tilde{f}_{3}=\frac{\delta_{\mathrm{t}_{1} \rho}^{2} \rho_{\mathrm{t}_{2} \mathrm{r}_{1}+\rho \mathrm{t}_{2}}^{2} \tilde{\mathrm{t}}_{1} \tilde{\mathrm{t}}_{1} \mathrm{r}_{1}}{K\left(\tilde{\delta}_{\mathrm{t}_{2} \mathrm{r}_{1}}^{2}+\rho \delta_{\mathrm{t}_{2}}^{2} \tilde{\delta}_{\mathrm{t}_{1} \mathrm{r}_{1}}^{2}\right)}$, with $\tilde{\delta}_{\mathrm{t}_{1}}^{2}=1+\delta_{\mathrm{t}_{1}}^{2}, \tilde{\delta}_{\mathrm{r}_{2}}^{2}=1+\alpha \delta_{\mathrm{r}_{1}}^{2}, \tilde{\delta}_{\mathrm{t}_{2} \mathrm{r}_{1}}^{2}=\rho \delta_{\mathrm{r}_{1}}^{2}+\delta_{\mathrm{t}_{2}}^{2}+$ 1 , and $\tilde{\delta}_{\mathrm{t}_{1} \mathrm{r}_{1}}^{2}=\delta_{\mathrm{r}_{1}}^{2}+\delta_{\mathrm{t}_{1}}^{2}+1$. For notation simplicity, we define $\tilde{\mathbf{K}}_{1}=\mathbf{H}_{2}\left(\mathbf{I}_{M}+\tilde{f}_{1} \mathbf{H}_{1} \mathbf{H}_{1}^{\mathrm{H}}\right) \mathbf{H}_{2}^{\mathrm{H}}$ and $\tilde{\mathbf{K}}_{2}=$ $\mathbf{H}_{2}\left(\mathbf{I}_{M}+\tilde{f}_{3} \mathbf{H}_{1} \mathbf{H}_{1}^{\mathrm{H}}\right) \mathbf{H}_{2}^{\mathrm{H}}$. Moreover, if $\mathbf{A}$ denotes a square matrix, then $[\mathbf{A}]_{k, k}$ expresses its $k$ th diagonal element.

In our analysis, we consider the values of $M, N$, and $K$ going to infinity with given ratios $\beta=\frac{K}{M}$ and $\gamma=\frac{N}{M}$. Subsequently, the average MMSE in 12 can be written as

$$
\mathrm{MMSE}_{\mathrm{avg}}=\lim _{K, M, N \rightarrow \infty} \mathbb{E}\left[\frac{1}{K} \operatorname{tr}\left\{\left(\mathbf{I}+\tilde{f}_{2} \tilde{\mathbf{K}}_{1}\right)^{-1}\left(\mathbf{I}+\tilde{f}_{2} \tilde{\mathbf{K}}_{2}\right)\right\}\right],
$$

where $\operatorname{tr}\{$.$\} denotes the trace operation. Using the trace$ inequality for the matrix product from [18] in (13), the lower bound for the average MMSE of the DH-AF MIMO relay system in the presence of residual additive transceiver can be expressed as

$$
\begin{aligned}
\operatorname{MMSE}_{\mathrm{avg}} & \geq \lim _{K, M, N \rightarrow \infty} \mathbb{E}\left[\frac{1}{K} \sum_{k=1}^{K} \frac{1+\tilde{f}_{2} \lambda_{K-k+1}\left(\tilde{\mathbf{K}}_{2}\right)}{1+\tilde{f}_{2} \lambda_{k}\left(\tilde{\mathbf{K}}_{1}\right)}\right] \\
& \rightarrow \int_{0}^{1} \frac{1+F_{\tilde{\mathbf{K}}_{2}}^{-1}(1-x)}{1+\tilde{f}_{2} F_{\tilde{\mathbf{K}}_{1}}^{-1}(x)} \mathrm{d} x
\end{aligned}
$$

where $\lambda_{i}(\mathbf{X})$ is the $i$ th ordered eigenvalue of matrix $\mathbf{X}$, and $F_{\mathbf{X}}^{-1}$ denotes the inverse of the asymptotic cumulative distribution function of $\mathbf{X}$. To compute this, we need to find the corresponding asymptotic probability density function (aepdf) i.e., $f_{\mathbf{X}}^{\infty}(x)$, which can be computed by using the following procedure.

For the simplicity of the analysis, we define the following notations

$$
\begin{aligned}
\tilde{\mathbf{M}}_{\bar{\alpha}} & =\mathbf{I}_{M}+\bar{\alpha} \mathbf{H}_{1} \mathbf{H}_{1}^{H} \\
\tilde{\mathbf{N}}_{1} & =\mathbf{H}_{1} \mathbf{H}_{1}^{\mathrm{H}} \\
\tilde{\mathbf{N}}_{2} & =\mathbf{H}_{2}^{\mathrm{H}} \mathbf{H}_{2} \\
\mathbf{K}_{\bar{\alpha}} & =\mathbf{H}_{2}^{\mathrm{H}} \mathbf{H}_{2}\left(\mathbf{I}_{M}+\bar{\alpha} \mathbf{H}_{1} \mathbf{H}_{1}^{H}\right)=\tilde{\mathbf{N}}_{2} \tilde{\mathbf{M}}_{\bar{\alpha}} \\
\tilde{\mathbf{K}}_{\bar{\alpha}} & =\mathbf{H}_{2}\left(\mathbf{I}_{M}+\bar{\alpha} \mathbf{H}_{1} \mathbf{H}_{1}^{\mathrm{H}}\right) \mathbf{H}_{2}^{\mathrm{H}}
\end{aligned}
$$

where $\bar{\alpha}=\tilde{f}_{1}$ for $\tilde{\mathbf{K}}_{1}$ and $\bar{\alpha}=\tilde{f}_{3}$ for $\tilde{\mathbf{K}}_{2}$. As noted in (14), we need the aepdf of $\tilde{\mathbf{K}}_{\bar{\alpha}}$ for our analysis. The relation between the aepdfs of $\mathbf{K}_{\bar{\alpha}}$ and $\mathbf{K}_{\bar{\alpha}}$ is given by the following proposition.

Proposition 1: The non-zero eigenvalues of $\mathbf{K}_{\bar{\alpha}}$ and $\tilde{\mathbf{K}}_{\bar{\alpha}}$ are identical.

Proof: See Appendix B.

Theorem 1: The Stieltjes transform $\mathcal{S}_{\mathbf{K}_{\tilde{\alpha}}}$ of the asymptotic distribution of eigenvalues of $\mathbf{K}_{\tilde{\alpha}}$ can be obtained for any $x \in \mathbb{C}$ by solving the following quartic polymonial

$$
\begin{array}{r}
\bar{\alpha}^{2} x^{2} \mathcal{S}_{\mathbf{K}_{\bar{\alpha}}}^{4} \\
+\left(2 \bar{\alpha}^{2}(1-\gamma) x+\bar{\alpha}^{2} x^{2}\right) S_{\mathbf{K}_{\bar{\alpha}}}^{3} \\
+\left(\bar{\alpha}^{2}(2-\beta-\gamma) x+\bar{\alpha}^{2}(\gamma-1)^{2}-\bar{\alpha} x\right) S_{\mathbf{K}_{\bar{\alpha}}}^{2} \\
+\left(\left(\bar{\alpha}^{2}(\beta(\gamma-1)-\gamma)+\bar{\alpha}(\gamma+\bar{\alpha}-x-1)\right) S_{\mathbf{K}_{\bar{\alpha}}}-\bar{\alpha}=0 .\right.
\end{array}
$$

Proof: See Appendix C.

The aepdf of $\mathbf{K}_{\bar{\alpha}}$ is then obtained by determining the imaginary part of the Stieltjes transform $\mathcal{S}_{\mathbf{K}_{\bar{\alpha}}}$ for real arguments as [19, Eq. 2.45]

$$
f_{\mathbf{K}_{\bar{\alpha}}}^{\infty}(x)=\lim _{y \rightarrow 0^{+}} \frac{1}{\pi} \operatorname{Im}\left\{\mathcal{S}_{\mathbf{K}_{\bar{\alpha}}}(x+\mathrm{j} y)\right\} .
$$

Subsequently, the aepdf of $\tilde{\mathbf{K}}_{a}$ is given by

$$
f_{\tilde{\mathbf{K}}_{\bar{\alpha}}}^{\infty}(x)=\left(1-\frac{1}{\gamma}\right)^{+} \delta(x)+\frac{1}{\gamma} f_{\mathbf{K}_{\bar{\alpha}}}^{\infty}(x) .
$$

\section{NUMERICAL RESUltS}

In this section, we verify the theoretical analysis carried out in previous sections and illustrate the impact of transceiver impairments on the MMSE filtering performance of DH-AF relay MIMO systems.

In order to validate our theoretical analysis, Fig. 1 provides the aepdf of $\tilde{\mathbf{K}}_{2}$, defined in Section III. In particular, the 
histogram represents the pdf of the matrix $\tilde{\mathbf{K}}_{2}$ calculated numerically based on Monte Carlo (MC) simulations. Furthermore, the solid line in the figure denotes the aepdf obtained by solving the polymonial (20) of the Stieltjes transform, and then by applying Lemma 4. From the result, we can observe a perfect agreement between theoretical analysis and MC simulations.

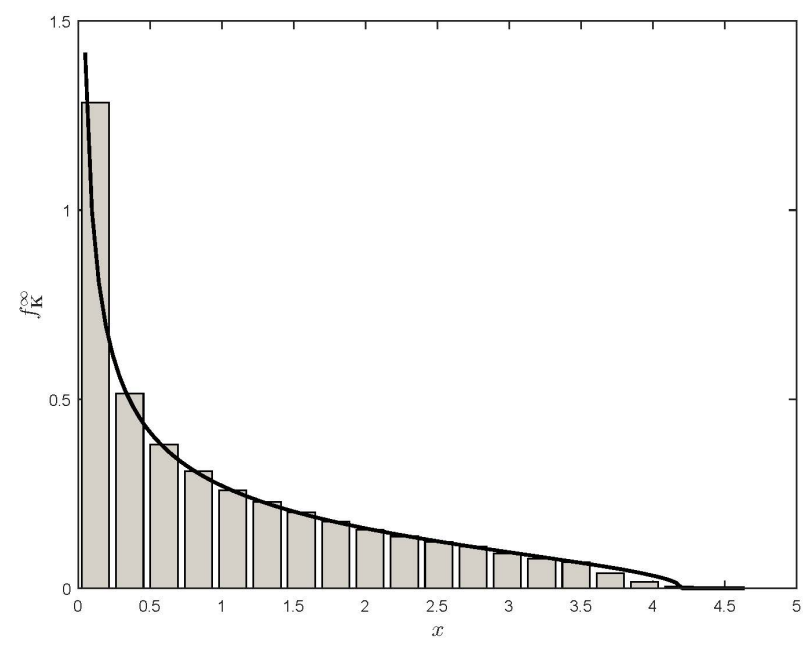

Fig. 1. Aepdf of $\tilde{\mathbf{K}}_{2}(\rho=20 \mathrm{~dB}, \alpha=2 \rho, \beta=1, \gamma=1, K=20$, $\left.M=20, N=20, \delta_{t_{1}}=\delta_{t_{2}}=\delta_{r_{1}}=\delta_{r_{2}}=0.08\right)$.

In Fig. 2, we plot the theoretical and simulated average MMSE versus the transmit SNR, i.e., $\rho$ for the following two cases: (i) without transceiver impairments, and (ii) with transceiver impairments on all nodes of the considered DHAF MIMO relay system. From the figure, we observe that the theoretical results provide tight lower bounds over the entire SNR range for all values of the transceiver impairments. Moreover, it can be noted that the average MMSE decreases with the increase in the value of $\rho$ in the absence of impairments, i.e., $\delta_{t_{1}}=\delta_{t_{2}}=\delta_{r_{1}}=\delta_{r_{2}}=0$, as expected. Whereas, the average MMSE first decreases with the increase in the value of $\rho$ and then saturates after a certain value of $\rho$. In addition, in Fig. 2 , we also show the effect of different values of impairments on the average MMSE. It can be depicted that the average MMSE decreases with the increase in the value of transceiver impairments. Another observation is that with the increase in the value of impairments, the saturation point appears earlier, i.e., at lower values of $\rho$.

Fig. 3 shows the MMSE performance versus the relay gain $\alpha$ by considering different values of impairments. From the figure, it can be noted that the theoretical lower bounds are tight over the considered range of $\alpha$. Furthermore, the average MMSE decreases monotonically with the value of $\alpha$ for the ideal case, whereas for the case with residual impairments, it first decreases and then starts to saturate after a certain value of $\alpha$ as for the case of $\rho^{2}$.

In order to illustrate the effect of different impairments on the MMSE filtering performance, we plot the average MMSE versus each node impairment in Fig. 4, by considering parameters $\rho=15 \mathrm{~dB}, N=40, M=10, K=20, \alpha=2 \rho$.

\footnotetext{
${ }^{2}$ In both Figs. 2 and 3, the saturation comes from the presence of the residual additive hardware impairments, while in the ideal case no saturation occurs [5]
}

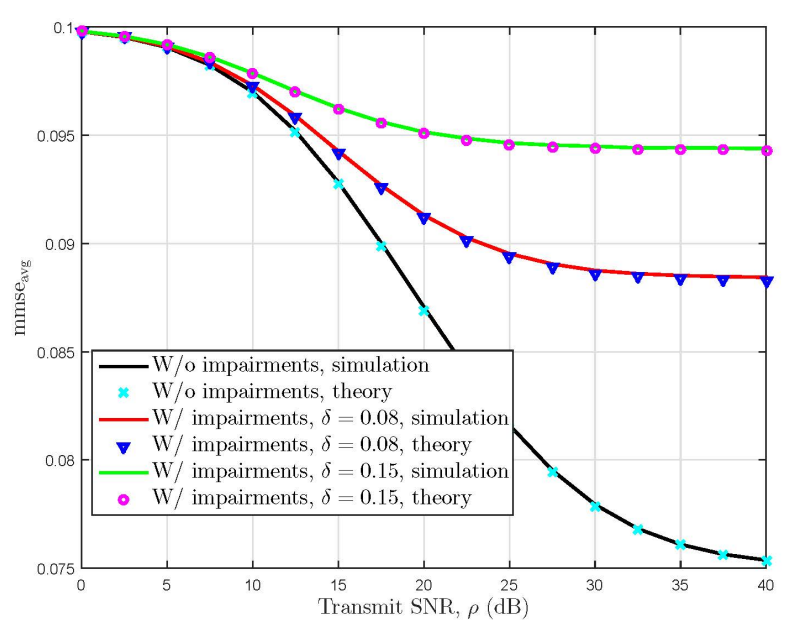

Fig. 2. Average MMSE versus $\rho(K=20, M=10, N=40, \alpha=2 \rho)$.

For this evaluation, all other impairments values are considered to be zero while examining the effect of a particular impairment. From the figure, it can be noted that the average MMSE increases with the increase in the value of impairment for all cases. Furthermore, the effect of $\delta_{r 2}$ on the MMSE performance is found to be the most severe as compared to the effects of other impairments, while the impairment $\delta_{t 1}$ has significantly less effect than the rest of the impairments.

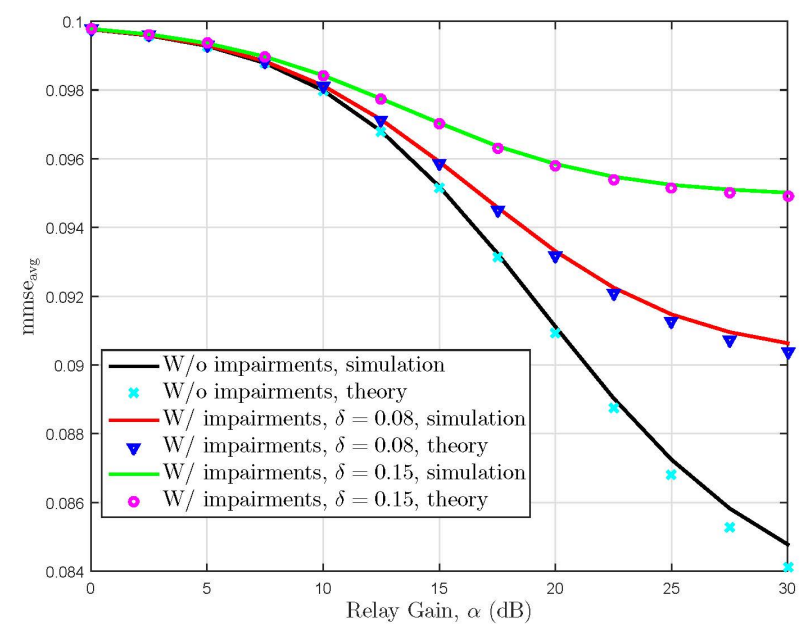

Fig. 3. Average MMSE versus $\alpha(\rho=15 \mathrm{~dB}, K=20, M=10, N=40$, $\alpha=2 \rho$ ).

\section{Conclusions}

In contrast to the most existing works with ideal transceiver hardware, this paper has studied the impact of additive residual transceiver impairments on the MMSE filtering performance of DH-AF massive MIMO relay channels. It was noted that the MMSE filtering performance of the considered system significantly degrades in the presence of residual additive hardware impairments. More importantly, it was shown that the average MMSE decreases with the increase in the transmit SNR and saturates after a certain value of SNR. In our future work, we plan to extend the current analysis to the case 


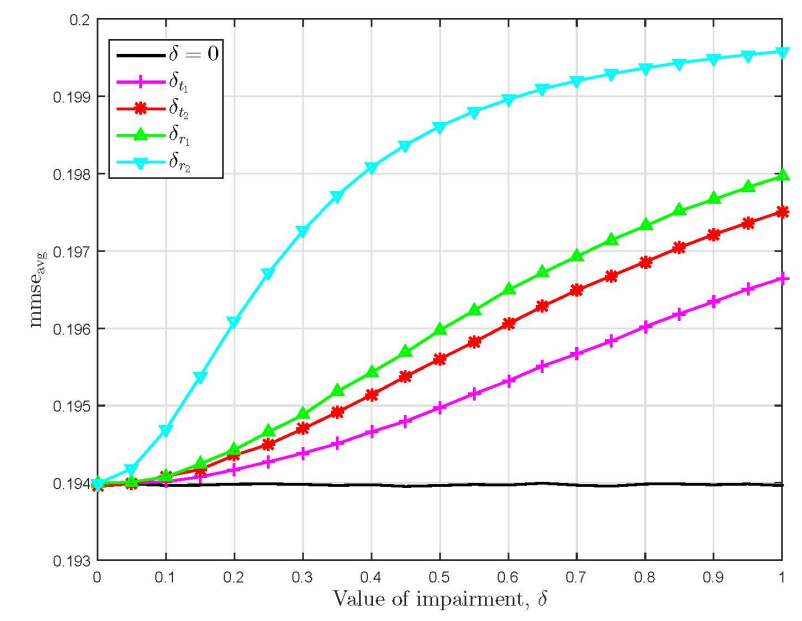

Fig. 4. Average MMSE versus $\delta(\rho=10 \mathrm{~dB}, K=20, M=10, N=40$, $\alpha=2 \rho)$.

of multiplicative impairments and also to study the MMSE filtering performance of other wireless channels such as multihop (more than two hops) relay channels and Rayleigh product MIMO channels.

\section{APPENDIX A}

\section{RANDOM MATRIX THEORY PRELIMINARIES}

Herein, we provide useful definitions and lemmas, related to the eigenvalue probability distribution function $f_{\mathbf{X}}(x)$ of a matrix $\mathbf{X}$, which are considered during our analysis ${ }^{3}$.

Definition 1 ( $\eta$-transform [19, Definition 2.11]): The $\eta$ transform of a positive semidefinite matrix $\mathbf{X}$ is defined as

$$
\eta_{\mathbf{X}}(\delta)=\int_{0}^{\infty} \frac{1}{1+\delta x} f_{\mathbf{X}}(x) \mathrm{d} x .
$$

Definition 2: [S-transform [19, Definition 2.15]] The Stransform of a positive semidefinite matrix $\mathbf{X}$ is defined as

$$
\Sigma_{\mathbf{X}}(x)=-\frac{x+1}{x} \eta_{\mathbf{X}}^{-1}(x+1) .
$$

Definition 3 (The Marčenko-Pastur law density function [20]): Given a Gaussian $M \times K$ channel matrix $\mathbf{H} \sim$ $\mathcal{C N}\left(\mathbf{0}, \frac{1}{M} \mathbf{I}_{M} \otimes \mathbf{I}_{K}\right)$, the aepdf of $\mathbf{H} \mathbf{H}^{\mathrm{H}}$ converges almost surely (a.s.) to the non-random limiting eigenvalue distribution of the Marčenko-Pastur law given by

$$
f_{\mathbf{H H}^{\mathrm{H}}}^{\infty}(x)=(1-\beta)^{+}(x)+\frac{\sqrt{(x-a)^{+}(b-x)^{+}}}{2 \pi x},
$$

where $a=(1-\sqrt{\beta})^{2}, b=(1+\sqrt{\beta})^{2}, \beta=\frac{K}{M}$, and $\delta(x)$ is Dirac's delta function.

Lemma 1 ( [19, Eqs. 2.87, 2.88]): The S-transform of the matrix $\mathbf{H}^{\mathrm{H}} \mathbf{H}$ is expressed as

$$
\Sigma_{\mathbf{H}^{\mathrm{H}} \mathbf{H}}(x, \beta)=\frac{1}{1+\beta x},
$$

while the S-transform of the matrix $\mathbf{H H}^{\mathrm{H}}$ is obtained as

$$
\Sigma_{\mathbf{H H}^{\mathrm{H}}}(x, \beta)=\frac{1}{\beta+x} .
$$

\footnotetext{
${ }^{3}$ Note that $\delta$ is a nonnegative real number.
}

Lemma 2 ( [19, Eq. 2.48]): The Stieltjes-transform of a positive semidefinite matrix $\mathbf{X}$ can be derived by its $\eta$-transform according to

$$
\mathcal{S}_{\mathbf{X}}(x)=-\frac{\eta_{\mathbf{X}}(-1 / x)}{x}
$$

\section{APPENDIX B}

\section{PROOF OF PREPOSITION 1}

Proof: Let us denote $\mathbf{A}=\mathbf{H}^{\mathrm{H}} \mathbf{H}$ and $\mathbf{B}=\mathbf{I}_{M}+\tilde{f}_{1} \mathbf{H}_{1} \mathbf{H}_{1}^{\mathrm{H}}$ Both matrices are Hermitian, as $\mathbf{B}$ is a sum of two Hermitians. Let us also define the EVD of $\mathbf{A}$ as $\mathbf{A}=\mathbf{U D U}^{H}$ with $\mathbf{C}=\mathbf{U D}^{1 / 2}$. Based on the Courant-Fischer minimax theorem, the $k$ th eigenvalue of $\mathbf{H B H}^{H}$ is given by

$$
\begin{array}{r}
\lambda_{K}\left(\mathbf{H B H}^{H}\right)=\min _{\alpha \in I_{k}} \max _{\mathbf{x} \in S_{k}^{\alpha} \backslash 0} \frac{\mathbf{x H B H} \mathbf{H}^{H} \mathbf{x}^{H}}{\mathbf{x} \mathbf{x}^{H}} \\
=\min _{\alpha \in I_{k}} \max _{\mathbf{x} \in S_{k}^{\alpha} \backslash 0} \frac{\mathbf{x} \mathbf{H H}^{H} \mathbf{x}}{\mathbf{x} \mathbf{x}^{H}} \frac{\mathbf{x H B} \mathbf{H}^{H} \mathbf{x}^{H}}{\mathbf{x H}(\mathbf{x H})^{H}} \\
=\min _{\alpha \in I_{k}} \max _{\mathbf{x} \in S_{k}^{\alpha} \backslash 0} \frac{\mathbf{x} \mathbf{x}^{H}}{\mathbf{x}\left(\mathbf{H H}^{H}\right)^{-1} \mathbf{x}^{H}} \frac{\mathbf{x B x}^{H}}{\mathbf{x x}^{H}} \\
=\min _{\alpha \in I_{k}} \max _{\mathbf{x} \in S_{k}^{\alpha} \backslash 0} \frac{\mathbf{x} \mathbf{A B} \mathbf{x}^{H}}{\mathbf{x} \mathbf{x}^{H}} \\
=\lambda_{K}(\mathbf{A B})
\end{array}
$$

The third step follows by evaluating over the span of the orthonormal eigenvectors of $\mathbf{C}^{-1}$.

\section{APPENDIX C \\ PROOF OF THEOREM 1}

Our aim is to obtain the Stieltjes transform of $\mathbf{K}_{\bar{\alpha}}$. As the first step, the following proposition provides the aepdf of $\tilde{\mathbf{M}}_{\bar{\alpha}}$.

Proposition 2: The aepdf of $\tilde{\mathbf{M}}_{\bar{\alpha}}$ converges almost surely to $(31)$.

Proof: By denoting $z$ and $x$ the eigenvalues of $\tilde{\mathbf{M}}_{\bar{\alpha}}$ and $\tilde{\mathbf{N}}_{1}$, respectively, the aepdf of $\tilde{\mathbf{M}}_{\bar{\alpha}}$ can be obtained after making the transformation $z(x)=(1+\bar{\alpha} x)$ as

$$
\begin{aligned}
f_{\tilde{\mathbf{M}}_{\bar{\alpha}}}^{\infty}(z) & =\left|\frac{1}{z^{\prime}\left(z^{-1}(x)\right)}\right| \cdot f_{\tilde{\mathbf{N}}_{1}}^{\infty}\left(z^{-1}(x)\right) \\
& =\frac{1}{\bar{\alpha}} f_{\tilde{\mathbf{N}}_{1}}^{\infty}\left(\frac{z-1}{\bar{\alpha}}\right)
\end{aligned}
$$

where the aepdf of $\tilde{\mathbf{N}}_{1}$ is given by (25).

The second step includes the derivation of $\eta_{\tilde{\mathbf{M}}_{\bar{\alpha}}}^{-1}(x)$, given by the next proposition.

Proposition 3: The inverse $\eta$-transform of $\tilde{\mathbf{M}}_{\bar{\alpha}}$ is given by (34).

Proof: Having obtained the aepdf of $\tilde{\mathbf{M}}_{\bar{\alpha}}$, use of Definition 1 allows to derive its $\eta$-transform as

$$
\eta_{\tilde{\mathbf{M}}_{\bar{\alpha}}}(\psi)=\int_{0}^{+\infty} \frac{1}{1+\psi x} f_{\tilde{\mathbf{M}}_{\bar{\alpha}}}^{\infty}(x) d x .
$$

If we make the necessary substitution, $\eta_{\tilde{\mathbf{M}}_{\bar{\alpha}}}(\psi)$ is written as in (33), which can be evaluated by performing certain substitutions as in [21]. Specifically, we set $x=w \bar{\alpha}+1, d x=\bar{\alpha} d w$, followed by $w=1+\beta+2 \sqrt{\beta} \cos \omega, d w=2 \sqrt{\beta}(-\sin \omega) d \omega$, and finally $\zeta=e^{i \omega}, d \zeta=i \zeta d \omega$. Hence, initially we calculate the poles $\zeta_{i}$ and residues $\rho_{i}$ of Eq. (33). Then, we perform 


$$
f_{\tilde{\mathbf{M}}_{\bar{\alpha}}}^{\infty}(x, \beta, \bar{\alpha}) \rightarrow \frac{\sqrt{(x-1-\bar{\alpha}+2 \bar{\alpha} \sqrt{\beta}-\bar{\alpha} \beta)(\bar{\alpha}+2 \bar{\alpha} \sqrt{\beta}+\bar{\alpha} \beta-x+1)}}{2 \bar{\alpha} \pi(x-1)} .
$$

$$
\eta_{\tilde{\mathbf{M}}_{\bar{\alpha}}}(\psi)=\frac{\bar{\alpha}}{4 i \pi} \oint_{|\zeta|=1} \frac{\left(\zeta^{2}-1\right)^{2}}{\zeta\left((1+\beta) \zeta+\sqrt{\beta}\left(\zeta^{2}+1\right)\right)\left(\zeta(1+\psi(1+\bar{\alpha}+\bar{\alpha} \beta))+\sqrt{\beta} \psi \bar{\alpha}\left(\zeta^{2}+1\right)\right)} d \zeta
$$

$$
\eta_{\tilde{\mathbf{M}}_{\bar{\alpha}}}^{-1}(x)=\frac{-x \bar{\alpha}-\beta \bar{\alpha}+\bar{\alpha}-1+\sqrt{x^{2} \bar{\alpha}^{2}+2 x \bar{\alpha}^{2} \beta-2 x \bar{\alpha}^{2}-2 x \bar{\alpha}+\beta^{2} \bar{\alpha}^{2}-2 \beta \bar{\alpha}^{2}+2 \beta \bar{\alpha}+\bar{\alpha}^{2}+2 \bar{\alpha}+1}}{2 x \bar{\alpha}} .
$$

an appropriate Cauchy integration by including the residues located within the unit disk. More concretely, we have

$$
\eta_{\tilde{\mathbf{M}}_{\bar{\alpha}}}(\psi)=-\frac{\beta}{2}\left(\rho_{0}+\rho_{2}+\rho_{4}\right),
$$

which after inversion results to (34).

Next, we provide the inverse $\eta$-transform of $\mathbf{K}_{\bar{\alpha}}$.

Proposition 4: The inverse $\eta$-transform of $\mathbf{K}_{\bar{\alpha}}$ is given by

$$
\eta_{\mathbf{K}_{\bar{\alpha}}}^{-1}(x)=\Sigma_{\tilde{\mathbf{N}}_{2}}(x-1) \eta_{\tilde{\mathbf{M}}_{\bar{\alpha}}}^{-1}(x)=\frac{1}{1+\gamma(x-1)} \eta_{\tilde{\mathbf{M}}_{\bar{\alpha}}}^{-1}(x) \text {. }
$$

Proof: The desired result is given by means of the free convolution

$$
\begin{gathered}
\Sigma_{\mathbf{K}_{\bar{\alpha}}}(x)=\Sigma_{\tilde{\mathbf{N}}_{\mathbf{2}}}(x) \Sigma_{\tilde{\mathbf{M}}_{\bar{\alpha}}}(x) \Longleftrightarrow \\
\left(-\frac{x+1}{x}\right) \eta_{\mathbf{K}_{\bar{\alpha}}}^{-1}(x+1)=\Sigma_{\tilde{\mathbf{N}}_{\mathbf{2}}}(x)\left(-\frac{x+1}{x}\right) \eta_{\tilde{\mathbf{M}}_{\bar{\alpha}}}^{-1}(x+1),
\end{gathered}
$$

where in (36) we have taken into advantage the asymptotic freeness between the deterministic matrix with bounded eigenvalues $\tilde{\mathbf{N}}_{2}$ and the unitarily invariant matrix $\tilde{\mathbf{M}}_{\bar{\alpha}}$ as well as we have applied Definition 2. By using 35 and with appropriate change of variables .e., $y=x+1$ provides (35).

The Stieltjes transform of $\mathbf{K}_{\bar{\alpha}}$ is the next required step for obtaining its aepdf. For this reason, we employ Lemma 2 to obtain the inverse of $\eta$-transform of $\mathbf{K}_{\bar{\alpha}}$ as

$$
x \eta_{\mathbf{K}_{\bar{\alpha}}}^{-1}\left(-x \mathcal{S}_{\mathbf{K}_{\bar{\alpha}}}(x)\right)+1=0 .
$$

Having calculated $\eta_{\mathbf{K}_{\bar{\alpha}}}^{-1}(x)$ from (35) we use (37), and after some algebraic manipulations, we obtain (20).

\section{ACKNOWLEDGEMENT}

This work was supported by a Marie Curie Intra European Fellowship within the 7th European Community Framework Programme for Research of the European Commission under grant agreements no. [330806], "IAWICOM", and partially by FNR, Luxembourg under the CORE projects "SeMIGod" and "SATSENT".

\section{REFERENCES}

[1] L. Le and E. Hossain, "Multihop cellular networks: Potential gains, research challenges, and a resource allocation framework," IEEE Communications Magazine, vol. 45, no. 9, pp. 66-73, September 2007.

[2] S. Sharma, M. Patwary, S. Chatzinotas, B. Ottersten, and M. AbdelMaguid, "Repeater for 5G wireless: A complementary contender for spectrum sensing intelligence," in IEEE International Conference on Communications (ICC), June 2015, pp. 1416-1421.
[3] S. Jin, M. R. McKay, C. Zhong, and K. K. Wong, "Ergodic capacity analysis of amplify-and-forward MIMO dual-hop systems," IEEE Trans. on Inform. Theory, vol. 56, no. 5, pp. 2204-2224, 2010.

[4] C.-K. Wen and K.-K. Wong, "On the sum-rate of uplink MIMO cellular systems with amplify-and-forward relaying and collaborative base stations," IEEE J. Sel. Areas Commun., vol. 28, no. 9, pp. 1409-1424, December 2010.

[5] S. Chatzinotas, "MMSE filtering performance of dual-hop amplify-andforward multiple-access channels," IEEE Wireless Commun. Lett., vol. 2, no. 1, pp. 122-125, 2013.

[6] J. Qi and S. Aïssa, "On the power amplifier nonlinearity in MIMO transmit beamforming systems," IEEE Trans. Commun., vol. 60, no. 3 , pp. 876-887, 2012.

[7] - "Analysis and compensation of I/Q imbalance in MIMO transmitreceive diversity systems," IEEE Trans. Commun., vol. 58, no. 5, pp. 1546-1556, 2010.

[8] G. Durisi, A. Tarable, C. Camarda, and G. Montorsi, "On the capacity of MIMO wiener phase-noise channels," in Information Theory and Applications Workshop (ITA), Feb. 2013, pp. 1-7.

[9] X. Zhang, M. Matthaiou, E. Björnson, M. Coldrey, and M. Debbah, "On the MIMO capacity with residual transceiver hardware impairments," in in Proc. IEEE Int. Conf. Commun. IEEE, 2014, pp. 5299-5305.

[10] E. Björnson, P. Zetterberg, M. Bengtsson, and B. Ottersten, "Capacity limits and multiplexing gains of MIMO channels with transceiver impairments," IEEE Commun. Lett., vol. 17, no. 1, pp. 91-94, 2013.

[11] E. Björnson, J. Hoydis, M. Kountouris, and M. Debbah, "Massive MIMO systems with non-ideal hardware: Energy efficiency, estimation, and capacity limits," IEEE Trans. Inform. Theory, vol. 60, no. 11, pp. 7112 7139, Nov 2014.

[12] F. Athley, G. Durisi, and U. Gustavsson, "Analysis of massive MIMO with hardware impairments and different channel models," arXiv preprint arXiv:1501.04200, 2015

[13] E. Björnson, M. Matthaiou, and M. Debbah, "A new look at dual-hop relaying: Performance limits with hardware impairments," IEEE Trans. Commun., vol. 61, no. 11, pp. 4512-4525, 2013.

[14] X. Xia, D. Zhang, K. Xu, W. Ma, and Y. Xu, "Hardware impairments aware transceiver for full-duplex massive mimo relaying," IEEE Trans. Signal Process., vol. 63, no. 24, pp. 6565-6580, Dec 2015.

[15] A. Papazafeiropoulos, S. Sharma, and S. Chatzinotas, "Impact of transceiver impairments on the capacity of dual-hop relay massive mimo systems," in Proc. of IEEE GLOBECOM 2015- Workshop on Massive MIMO: From theory to practice, Dec. 2015.

[16] T. Schenk, RF imperfections in high-rate wireless systems: impact and digital compensation. Springer Science \& Business Media, 2008.

[17] C. Studer, M. Wenk, and A. Burg, "MIMO transmission with residual transmit-rf impairments," in ITG/IEEE Work. Smart Ant. (WSA). IEEE, 2010, pp. 189-196.

[18] J. B. Lasserre, "A trace inequality for matrix product," IEEE Transactions on Automatic Control, vol. 40, no. 8, pp. 1500-1501, Aug 1995.

[19] A. M. Tulino and S. Verdú, Random matrix theory and wireless communications. Now Publishers Inc., 2004, vol. 1, no. 1.

[20] J. W. Silverstein and Z. Bai, "On the empirical distribution of eigenvalues of a class of large dimensional random matrices," Journal of Multivariate analysis, vol. 54, no. 2, pp. 175-192, 1995.

[21] Z. Bai and J. W. Silverstein, Spectral analysis of large dimensional random matrices. Springer, 2010, vol. 20. 